ARTÍ́CULO ORIGINAL

\title{
Uso de residuos de procesos de transformación secundaria de tres especies tropicales en la fabricación de paneles encolados lateralmente
}

\author{
Use of residues from secondary transformation processes of three tropical species \\ in the manufacture of Edge glued panels

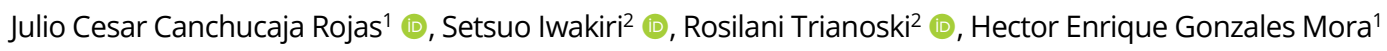 \\ ${ }^{1}$ Universidad Nacional Agraria La Molina - UNALM, Molina, Peru \\ ${ }^{2}$ Universidade Federal do Paraná - UFPR, Curitiba, PR, Brasil
}

Como citá: Rojas, J. C. C., Iwakiri, S., Trianoski, R., \& Mora, H. E. G. (2020). Uso de residuos de procesos de transformación secundaria de tres especies tropicales en la fabricación de paneles encolados lateralmente. Scientia Forestalis, 48(125), e3168. https://doi.org/10.18671/scifor.v48n125.20

\begin{abstract}
Resumen
Este estudio tuvo como objetivo evaluar la viabilidad de fabricar paneles encolados lateralmente (EGP), a partir de residuos de madera provenientes de procesos de trasformación secundaria de las especies tropicales Cariniana domestica (Mart.) Miers (Cachimbo), Copaifera paupera (Copaiba), Cedrelinga cateniformis (Ducke) Ducke (tornillo) y sus combinaciones. Se evaluó la calidad de las juntas encoladas con los adhesivos acetato de polivinilo (PVA) y emulsión polimérica de isocianato (EPI) con un gramaje de $180 \mathrm{~g} / \mathrm{m}^{2}$, mediante el ensayo de cizallamiento en la línea de cola, utilizando las normas UNE-EN 13353 y UNE-EN 13354. Los resultados de los ensayos sin pretratamiento de todas las juntas encoladas estudiadas con los adhesivos PVA y EPI, demostraron su viabilidad técnica para la manufactura de paneles EGP para uso en interiores, recomendando el empleo del adhesivo PVA para reducir costos. Los resultados de los ensayos de las juntas encoladas con adhesivo EPI de las especies Cariniana domestica, Copaifera paupera y la combinación Cariniana domestica/Copaifera paupera mostraron aptitud para la producción de paneles EGP para uso en ambiente seco y húmedo, mientras que los elaborados con la especie Cedrelinga cateniformis y la combinación Copaifera paupera/Cedrelinga cateniformis para uso en ambiente seco.
\end{abstract}

Palabras-clave: Residuos de madera; Maderas tropicales; Paneles EGP; Adhesivo PVA; Adhesivo EPI.

\begin{abstract}
The objective of this study was to evaluate the feasibility of manufacturing edge glued panels (EGP), made with wood waste from secondary transformation processes of the tropical species Cariniana domestica (Mart.) Miers (Cachimbo), Copaifera paupera (Copaiba), Cedrelinga cateniformis (Ducke) Ducke (screw) and their combinations. The quality of the joints glued with polyvinyl acetate (PVA) and isocyanate polymer emulsion (EPI) adhesives with a grammage of $180 \mathrm{~g} / \mathrm{m}^{2}$ was evaluated through glue line shear test using the UNE-EN standards 13353 and UNE-EN 13354. Tests without pretreatment results for all the glued joints with PVA and EPI adhesives, demonstrated their technical feasibility for manufacturing EGP panels for indoor use, being recommendable to use PVA adhesive to reduce costs. Joints glued with EPI adhesive in the species Cariniana domestica, Copaifera paupera and the combination Cariniana domestica / Copaifera paupera proved their aptitude for the production of EGP panels for use in dry and humid environment; while those elaborated with Cedrelinga cateniformis and the combination Copaifera paupera / Cedrelinga cateniformis for use in a dry environment.
\end{abstract}

Keywords: Wood residues; Tropical timber; Edge glued panel; PVA adhesive; EPI adhesive.

Fuente de financiamiento: Ninguna.

Conflicto de interés: nada que declarar.

Autor correspondiente: setsuo.ufpr@gmail.com

Recibido: 19 octubre 2018.

Aprobado: 26 junio 2019.

Editor: Francides Gomes da Silva Júnior.

(c) Este es un artículo publicado en acceso abierto (Open Access) bajo la licencia Creative Commons Attribution, que permite su uso, distribución y reproducción en cualquier medio, sin restricciones siempre que el trabajo original sea debidamente citado. 


\section{INTRODUCCIÓN}

Las industrias de transformación secundaria de la madera en el Perú, entre las cuales se encuentran las fábricas de mobiliario escolar, generan actualmente grandes cantidades de residuos, debido mayormente al uso de materia prima de calidad irregular y tecnologías inadecuadas de transformación. Estos residuos, en el mejor de los casos se utilizan como fuente de energía, o bien se convierten en desechos, que ocasionan contaminación ambiental y gastos para su eliminación de la planta.

De acuerdo a Held et al. (2015), las principales especies maderables utilizadas para la fabricación de mobiliario escolar, que son adquiridos en grandes cantidades por instituciones públicas del Perú, son la Cariniana domestica (Mart.) Miers (cachimbo), Copaifera paupera (copaiba) y Cedrelinga cateniformis (Ducke) Ducke (tornillo); los cuales producen grandes volúmenes de residuos durante su procesamiento.

Una alternativa de aprovechamiento de estos residuos es en la fabricación de paneles EGP, actualmente utilizados en la industria del mueble y en carpintería de obra. El panel EGP es definido como un conjunto de listones de madera encolados lateralmente y en los extremos mediante juntas "finger joint", elaboradas del mismo tipo de madera (Iwakiri, 2005).

En el mercado peruano se están utilizando paneles EGP encolados mayormente con adhesivos PVA, y en menor proporción con EPI, empalmados con juntas tipo "finger joint", los cuales mayormente se están importando de Chile, Brasil y Ecuador, y en menor cantidad son producidos en el país, utilizando las especies tropicales Dypterix micrantha, Cedrela odorata, Simarouba amara, entre otras, y la Guazuma crinita proveniente de plantaciones.

El adhesivo PVA es un producto obtenido por la polimerización en medio acuoso del acetato de vinilo, conocido como "cola blanca", muy resistente mecánicamente en ambiente seco, aunque muy rígida y poco resistente a la humedad, y es utilizado en la manufactura de paneles EGP (Iwakiri, 2005; Frihart \& Hunt, 2010). Por otro lado, el adhesivo EPI, emulsión polimérica de isocianato, está compuesto por una base de poliacetato de vinilo y un isocianato polimérico cuya función es catalizar la reacción del curado del adhesivo, que se produce por medio de la reacción de los grupos isocianatos con los grupos hidroxilos de la madera; muestra además una alta resistencia al agua y temperatura, buena estabilidad, curado rápido, flexible, pero que tiene como desventaja su mayor costo y se deben mezclar dos componentes antes de utilizarse (Frihart \& Hunt, 2010; Frihart, 2013).

Almeida (2015) indica que la masa especifica de la madera, el contenido de humedad y el coeficiente de anisotropía son las propiedades físicas más relevantes de la madera a utilizarse en la fabricación de paneles EGP. Del mismo modo, la masa especifica es la propiedad que más influye en sus propiedades físicas y mecánicas. La cantidad y distribución de los vasos de la madera influyen, durante el encolado de la madera, en su habilidad de absorber agua u otros solventes de los adhesivos (Pizzi \& Mittal, 2003).

Iwakiri (2005) y Frihart \& Hunt (2010) mencionan que la porosidad de la madera tiene una relación inversa con su masa especifica en la facilidad de la penetración del adhesivo en la estructura leñosa; por ello, en maderas de masa especifica alta, al poseer menos espacios vacíos, dificultan la penetración del adhesivo hacia las capas interiores, ocasionando una baja adhesión mecánica.

La cantidad de extractivos y el pH de la madera, así como las condiciones de la superficie a ser encolada, pueden perjudicar su humedecimiento por el adhesivo, dificultando la penetración en la superficie submicroscópica de la madera. El grado de pH de la madera se ubica en un rango de 3 a 6, pudiendo ocurrir cambios dentro de una pieza de madera en función a la migración de los extractivos de las capas más internas hacia las capas superficiales, alterando las condiciones del encolado (Iwakiri, 2005).

Este trabajo tiene como objetivo determinar la viabilidad tecnológica para la producción de paneles EGP, con residuos de madera de tres especies tropicales provenientes de procesos de transformación secundaria, para lo cual se evaluó la resistencia al cizallamiento de las juntas encoladas de las maderas estudiadas y sus combinaciones, utilizando dos tipos de adhesivos y tres pretratamientos. 


\section{MATERIAL Y MÉTODOS}

En la presente investigación se utilizaron residuos de madera secas al horno de las especies tropicales Cedrelinga cateniformis, Cariniana domestica y Copaifera paupera, procedentes del Departamento de Ucayali, Perú, colectados de los procesos de fabricación de mobiliario escolar de las empresas KFR Ltda. e Industry of furniture S.A. ubicadas en el Distrito de San Juan de Lurigancho, Departamento de Lima, Perú. Para el encolado de las piezas se utilizaron los adhesivos PVA 3010 y el EPI 1911 con el componente HARDENER 1999, fabricado por la empresa Akzo Nobel Perú S.A.C.

Los residuos usados fueron piezas de madera de 2 a $4 \mathrm{~cm}$ de espesor y de 5 a $30 \mathrm{~cm}$ de ancho, que presentaron dimensiones o defectos no permitidos para la fabricación de mobiliario escolar, de los cuales se obtuvieron listones de $20 \times 50 \times 300 \mathrm{~mm}$ de espesor, ancho y largo, eliminándose los defectos presentes.

La determinación de la masa especifica aparente de la madera se efectuó en base a lo señalado en la NTP 250.11 (Instituto Nacional de la Calidad, 2014b) y el contenido de humedad con la NTP 2151.010 (Instituto Nacional de la Calidad, 2014a). Las propiedades químicas fueron determinadas a partir de astillas obtenidas de las maderas en estudio, cuantificando los extractivos totales con la norma TAPPI T 204 (Thechnical Association of the Pulp and Paper Industry, 2017) y el pH de la madera con la TAPPI T 252 (Thechnical Association of the Pulp and Paper Industry, 2016).

Las juntas fueron elaboradas con listones encolados lateralmente en pares, de acuerdo a los tratamientos en estudio (especie y/o combinación, adhesivo). El adhesivo fue aplicado en la cara lateral de uno de los listones que conforma el par con una espátula de metal y con un gramaje de $180 \mathrm{~g} / \mathrm{m}^{2}$ para ambos adhesivos estudiados. De acuerdo a las indicaciones del fabricante del adhesivo, los pares de listones encolados fueron prensados en una prensa fría a temperatura ambiente, con un tiempo de prensado de 60 minutos y aplicando una presión de $8 \mathrm{kgf} / \mathrm{cm}^{2}$. Se efectuó el prensado de juntas encoladas para cada tipo de adhesivo, entre listones de la misma especie y con las combinaciones mostradas en la Tabla 1.

Tabla 1. Diseño experimental.

\begin{tabular}{cccc}
\hline Tratam. & Especie /combinaciones & Adhesivo. & Pretratam. \\
\hline 1 & Cariniana domestica & $\mathrm{PVA}$ & $\mathrm{S}^{1} / \mathrm{AS}^{2} / \mathrm{AH}^{3}$ \\
2 & Copaifera paupera & $\mathrm{PVA}$ & $\mathrm{S}^{1} / \mathrm{AS}^{2} / \mathrm{AH}^{3}$ \\
3 & Cedrelinga cateniformis & $\mathrm{PVA}$ & $\mathrm{S}^{1} / \mathrm{AS}^{2} / \mathrm{AH}^{3}$ \\
4 & Cariniana domestica/Copaifera paupera & $\mathrm{PVA}$ & $\mathrm{S}^{1} / \mathrm{AS}^{2} / \mathrm{AH}^{3}$ \\
5 & Cariniana domestica/Cedrelinga cateniformis & $\mathrm{PVA}$ & $\mathrm{S}^{1} / \mathrm{AS}^{2} / \mathrm{AH}^{3}$ \\
6 & Copaifera paupera/Cedrelinga cateniformis & $\mathrm{PVA}$ & $\mathrm{S}^{1} / \mathrm{AS}^{2} / \mathrm{AH}^{3}$ \\
7 & Cariniana domestica & $\mathrm{EPI}$ & $\mathrm{S}^{1} / \mathrm{AS}^{2} / \mathrm{AH}^{3}$ \\
8 & Copaifera paupera & $\mathrm{EPI}$ & $\mathrm{S}^{1} / \mathrm{AS}^{2} / \mathrm{AH}^{3}$ \\
9 & Cedrelinga cateniformis & $\mathrm{EPI}$ & $\mathrm{S}^{1} / \mathrm{AS}^{2} / \mathrm{AH}^{3}$ \\
10 & Cariniana domestica/Copaifera paupera & $\mathrm{EPI}$ & $\mathrm{S}^{1} / \mathrm{AS}^{2} / \mathrm{AH}^{3}$ \\
11 & Cariniana domestica/Cedrelinga cateniformis & $\mathrm{EPI}$ & $\mathrm{S}^{1} / \mathrm{AS}^{2} / \mathrm{AH}^{3}$ \\
12 & Copaifera paupera/Cedrelinga cateniformis & $\mathrm{EPI}$ & $\mathrm{S}^{1} / \mathrm{AS}^{2} / \mathrm{AH}^{3}$ \\
\hline
\end{tabular}

Pretratamiento: (') Sin pretratamiento; ( $\left.{ }^{2}\right)$ SWP/1 Ambiente seco; ( $\left.{ }^{3}\right)$ SWP/2 Ambiente húmedo.

Las juntas encoladas y prensadas fueron acondicionadas en una cámara de climatización a una temperatura de $20 \pm 3^{\circ} \mathrm{C}$ y humedad relativa de $65 \pm 5 \%$, luego del cual se confeccionaron las probetas para los ensayos de cizallamiento en la línea de cola. Se prepararon 30 probetas por tratamiento, de las cuales 10 fueron ensayados sin pretratamiento, 10 con pretratamiento para ambiente seco y 10 para ambiente húmedo. Para el pretratamiento en ambiente seco, las probetas fueron sumergidas en agua a una temperatura de $20^{\circ} \mathrm{C} \pm 3^{\circ} \mathrm{C}$ por 24 horas antes de los ensayos; mientras que, para el pretratamiento en ambiente húmedo, fueron sometidas durante 6 horas en agua hirviendo y luego a un enfriamiento por al menos 1 hora en agua a una 
temperatura de $20 \pm 3^{\circ} \mathrm{C}$, de acuerdo a la norma UNE-EN 13354 (Asociacion Española de Normalizacion y Certificacion, 2009).

Los ensayos fueron efectuados de acuerdo a los procedimientos descritos en la norma UNE-EN 13354 (Asociacion Española de Normalizacion y Certificacion, 2009) y los valores obtenidos se compararon con los requisitos indicados en la norma UNE-EN 13353 (Asociacion Española de Normalizacion y Certificacion, 2011), para lo cual se determinó el $5^{\circ}$ percentil inferior de la resistencia al cizallamiento de las probetas ensayadas con el procedimiento señalado en la norma UNE-EN 326-1 (Asociacion Española de Normalizacion y Certificacion, 1995); realizándose además la determinación de los porcentajes de falla de la madera, en base a la norma UNE-EN 314-1 (Asociacion Española de Normalizacion y Certificacion, 2007).

Para las características físicas y químicas se calculó el promedio y coeficiente de variación por especie, realizándose además un ANOVA para la comparación de medias y una prueba de Tukey, con un nivel de confianza de 95\%, en los casos que se encontraron diferencias significativas en al menos una media. Para los resultados de cizallamiento se calculó el valor promedio, el $5^{\circ}$ percentil inferior y el coeficiente de variabilidad, mientras que para el porcentaje de falla por la madera se calculó el valor promedio. Para cada pretratamiento se realizó un ANOVA aplicado a un diseño factorial (factores: Especies encoladas y tipo de adhesivo) y una prueba de Tukey con un nivel de confianza de 95\%, empleando el programa estadístico R Core Team v.3.5.0, en los casos que existieron diferencias significativas en al menos una media.

\section{RESULTADOS Y DISCUSION}

\section{Propiedades de la madera}

Los resultados de la masa específica aparente, contenido de humedad, extractivos totales y $\mathrm{pH}$ de las tres maderas estudiadas se muestran en la Tabla 2.

Tabla 2. Valores medios y coeficientes de variación de las propiedades de la madera.

\begin{tabular}{cccc}
\hline \multirow{2}{*}{ Característica } & \multicolumn{3}{c}{ Especies } \\
\cline { 2 - 4 } & Cariniana domestica & Copaifera paupera & Cedrelinga cateniformis \\
\hline Masa especifica aparente & $0,76^{\mathrm{a}}(3,67)$ & $0,72^{\mathrm{a}}(7,89)$ & $0,59^{\mathrm{b}}(3,92)$ \\
(g/cm $\left.{ }^{3}\right)$ & $11,65^{\mathrm{a}}(5,33)$ & $11,66^{\mathrm{a}}(5,28)$ & $11,62^{\mathrm{a}}(2,27)$ \\
Contenido de humedad $(\%)$ & $4,07^{\mathrm{b}}(17,51)$ & $7,01^{\mathrm{a}}(7,43)$ & $4,30^{\mathrm{b}}(4,27)$ \\
Extractivos totales $(\%)$ & $5,19^{\mathrm{b}}(0,68)$ & $5,98^{\mathrm{a}}(1,97)$ & $5,14^{\mathrm{b}}(1,01)$ \\
\hline $\mathrm{pH}$ & & &
\end{tabular}

Las medias seguidas de una misma letra en una misma fila son estadísticamente iguales, por la prueba de Tukey al 95\% de probabilidad. Los valores entre paréntesis son los coeficientes de variación en porcentaje.

Las especies Cariniana domestica $\left(0,76 \mathrm{~g} / \mathrm{cm}^{3}\right)$ y Copaifera paupera $\left(0,72 \mathrm{~g} / \mathrm{cm}^{3}\right)$ presentaron mayores valores promedio de masa especifica aparente que la Cedrelinga cateniformis $\left(0,59 \mathrm{~g} / \mathrm{cm}^{3}\right)$; asimismo, de acuerdo a lo señalado en la norma UNE 56-540 (Asociacion Española de Normalizacion y Certificacion, 1978), las especies Copaifera paupera y Cedrelinga cateniformis son clasificadas como semipesadas, mientras que la Cariniana domestica como pesada. La prueba de Tukey, con un nivel de confianza de 95\%, muestra que la masa especifica aparente de la Cariniana doméstica y Copaifera paupera no presentan diferencias significativas entre sí, mientras que ambas sí tienen diferencias significativas con la Cedrelinga cateniformis.

El contenido de humedad promedio de las tres maderas fluctúan de 11,62\% a 11,66\%, y que se ubican dentro de los rangos indicados por el fabricante de los adhesivos PVA y EPI, que son de $8 \%$ a $14 \%$ y de $8 \%$ a $15 \%$ respectivamente; encontrándose además que no existen diferencias significativas entre las tres especies estudiadas. 
Con respecto a las propiedades químicas, se encontró que el mayor valor promedio de extractivos totales obtenido fue en la Copaifera paupera con 7,01\%, mientras que para la Cariniana doméstica y Cedrelinga cateniformis fueron de 4,07\% y 4,30\% respectivamente.

Las especies Cariniana doméstica y Cedrelinga cateniformis no difieren estadísticamente entre sí, pero sí existen diferencias significativas con respecto a la Copaifera paupera. Los valores promedio de extractivos totales obtenidos de las tres especies estudiadas se encuentran dentro del rango de 1\% a 10\% que reporta Gonzales (2013) para maderas tropicales, por lo que los porcentajes reportados no deberían producir problemas de interacción del adhesivo y la madera.

Los valores promedio de $\mathrm{pH}$ hallados en las tres especies fluctúan de 5,14 a 5,98, no encontrándose diferencias significativas entre la Cariniana domestica y Cedrelinga cateniformis, pero sí existen diferencias significativas al compararlas con la Copaifera paupera. Los resultados obtenidos se encuentran dentro del rango de 3 a 6 indicado por Iwakiri (2005); quien además señala que el pH y los extractivos presentes en la madera pueden inhibir las reacciones químicas del fraguado de la cola, disminuyendo la resistencia y adhesión adecuada en la línea de cola.

\section{Resistencia de las juntas encoladas al ensayo de cizallamiento- sin pretratamiento}

Los resultados de los valores promedio de cizallamiento, porcentaje de falla en la madera y el $5^{\circ}$ percentil inferior de las juntas encoladas en las probetas ensayadas sin pretratamiento se muestran en la Tabla 3.

Tabla 3. Resultados de los ensayos de cizallamiento sin pretratamiento/interacciones.

\begin{tabular}{|c|c|c|c|}
\hline Tratamiento & Cizallamiento (MPa) & Falla (\%) & $\begin{array}{c}5^{\circ} \text { Percentil inferior } \\
(\mathrm{MPa})\end{array}$ \\
\hline PVA & $9,10 b(2,64)$ & & \\
\hline EPI & $10,00 \mathrm{a}(2,40)$ & & \\
\hline 1. CA $\times$ PVA & $9,35^{b}(19,60)$ & 80,0 & 6,84 \\
\hline 2. CO x PVA & $8,84^{\mathrm{b}}(24,03)$ & 81,5 & 6,31 \\
\hline 3. TO x PVA & $9,03^{b}(18,31)$ & 78,0 & 6,96 \\
\hline 4. CA/CO x PVA & $9,24^{\mathrm{b}}(16,09)$ & 76,0 & 7,21 \\
\hline 5. CA/TO x PVA & $9,42^{\mathrm{b}}(21,03)$ & 77,0 & 7,17 \\
\hline 6. CO/TO x PVA & $8,72^{\mathrm{b}}(20,48)$ & 62,0 & 6,25 \\
\hline 7. CA $\times$ EPI & $10,53^{\mathrm{a}}(20,18)$ & 77,5 & 7,49 \\
\hline 8. CO x EPI & $10,15^{\mathrm{a}}(16,56)$ & 82,0 & 7,90 \\
\hline 9. TO x EPI & $9,77^{\mathrm{a}}(19,77)$ & 86,5 & 7,16 \\
\hline 10. CA/CO x EPI & $9,82^{\mathrm{a}}(23,62)$ & 83,5 & 7,13 \\
\hline 11. CA/TO x EPI & $10,04^{\mathrm{a}}(11,02)$ & 92,0 & 8,93 \\
\hline 12. CO/TO x EPI & $9,73^{a}(20,61)$ & 90,5 & 7,55 \\
\hline FV. & & $\mathrm{F}$ & $p$-value \\
\hline Especie & & 0,36 & 0,871 \\
\hline Adhesivo & & 7,20 & 0,009 \\
\hline Especie:adhesivo & & 0,13 & 0,984 \\
\hline
\end{tabular}

CA: Cariniana domestica; CO: Copaifera paupera; TO: Cedrelinga cateniformis; FV: Fuente de variación. Las medias seguidas de una misma letra en una misma columna son estadísticamente iguales, por la prueba de Tukey al $95 \%$ de probabilidad. Los valores entre paréntesis son los coeficientes de variación en porcentaje.

Los valores promedio de resistencia al cizallamiento de las juntas encoladas con adhesivo PVA fluctuaron de 8,72 MPa (Copaifera paupera/Cedrelinga cateniformis) a 9,42 MPa (Cariniana domestica/Cedrelinga cateniformis), mientras que para el adhesivo EPI fueron de 9,73 MPa (Copaifera paupera/Cedrelinga cateniformis) a 10,53 MPa (Cariniana domestica).

Los resultados del análisis de variancia muestran que el efecto de las especies empleadas no es significativo sobre la resistencia al cizallamiento. Por otro lado, el adhesivo utilizado sí presenta un efecto significativo, con un nivel de confianza de 95\%, encontrándose 
que los ensayos con el adhesivo EPI mostraron una mayor resistencia (10,00 $\mathrm{MPa})$, al compararlas con los efectuados con el adhesivo PVA (9,10 MPa).

Los valores obtenidos para todas las juntas encoladas con los adhesivos PVA y EPI fueron inferiores a los reportados por Iwakiri et al. (2016) para las especies tropicales Protium puncticulatum y dinizia excelsa, con masas específicas aparentes de $1,01 \mathrm{~g} / \mathrm{cm}^{3} \mathrm{y}$ 0,88 g/ $\mathrm{cm}^{3}$, encontrándose valores de 16,56 y 15,07 MPa para el adhesivo PVA y de 17,73 y 15,30 MPa para el adhesivo EPI, pero similares a los encontrados por Iwakiri et al. (2015) para las especies Cryptomeria japónica y Sequoia sempervirens, con masas especificas aparentes de 0,43 $\mathrm{g} / \mathrm{cm}^{3}$ y 0,50 $\mathrm{g} / \mathrm{cm}^{3}$, alcanzando valores de 8,04 y 9,03 MPa para el adhesivo PVA y de 8,16 y 10,51 MPa para el adhesivo EPI.

Los porcentajes de falla en la madera fluctuaron de 62,0\% a $81,5 \%$ para las juntas encoladas con el adhesivo PVA, y de 77,0\% a 92,0\% con el adhesivo EPI, siendo mayores a los obtenidos por Iwakiri et al. (2016) para las especies Protium puncticulatum y dinizia excelsa con los adhesivos PVA (14,5\% y 44,1\%) y EPI (46,7\% y 82,7\%), y similares a los reportados por Iwakiri et al. (2015) para las especies Cryptomeria japónica y Sequoia sempervirens, con 71,0\% y $64,0 \%$ para el adhesivo PVA y de $87,5 \%$ y $72,5 \%$ para el adhesivo EPI. Las diferencias encontradas con los resultados de las especies Protium puncticulatum y dinizia excelsa pueden deberse a que presentan masas especificas mayores a las ensayadas en el presente estudio, y han fallado mayormente en la madera, por lo que tuvieron mayor resistencia mecánica al ensayo de cizallamiento.

Los valores promedio obtenidos del $5^{\circ}$ percentil inferior para las juntas encoladas con Ios adhesivos PVA y EPI variaron de 6,25 MPa a 8,93 MPa. Los resultados muestran que todos los tratamientos evaluados con los dos adhesivos utilizados cumplen con los requisitos mínimos del $5^{\circ}$ percentil inferior de la resistencia al cizallamiento, para el que la norma UNE-EN 13353 (Asociacion Española de Normalizacion y Certificacion, 2011) indica un valor mínimo de 2,5 MPa. Se debe considerar que este requisito solo es aplicable para evaluar las juntas encoladas ensayadas luego del pretratamiento adecuado para su uso en ambiente seco, húmedo o exterior.

Resistencia de las juntas encoladas al ensayo de cizallamiento- con pretratamiento ambiente seco

La Tabla 4 muestra los resultados de los ensayos de cizallamiento, porcentaje de falla en la madera y el $5^{\circ}$ percentil inferior de las juntas encoladas con pretratamiento en ambiente seco.

Tabla 4. Resultados de los ensayos de cizallamiento con pretratamiento ambiente seco/interacciones.

\begin{tabular}{|c|c|c|c|}
\hline Tratamiento & Cizallamiento (MPa) & Falla (\%) & $5^{\circ}$ Percentil inferior ( $\left.\mathrm{MPa}\right)$ \\
\hline 1. CA X PVA & $0,82^{\mathrm{e}}(36,36)$ & 0 & 0,49 \\
\hline 2. CO x PVA & $1,28^{\mathrm{cd}}(11,44)$ & 0 & 1,12 \\
\hline 3. TO x PVA & $1,69^{c}(23,32)$ & 0 & 1,26 \\
\hline 4. $C A / C O \times P V A$ & $0,80^{e}(33,69)$ & 0 & 0,42 \\
\hline 5. CA/TO x PVA & $0,81^{\mathrm{e}}(31,38)$ & 0 & 0,50 \\
\hline 6. CO/TO $\times$ PVA & $0,98^{\text {de }}(25,36)$ & 0 & 0,67 \\
\hline 7. CA $\times$ EPI & $5,19^{\mathrm{ab}}(11,59)$ & 14,5 & 4,22 \\
\hline 8. CO x EPI & $5,23^{\mathrm{ab}}(18,71)$ & 21,5 & 3,87 \\
\hline 9. TO $\times$ EPI & $4,31^{\mathrm{ab}}(22,94)$ & 12,5 & 3,17 \\
\hline 10. CA/CO $\times \mathrm{EPI}$ & $3,76^{b}(39,09)$ & 7,0 & 2,06 \\
\hline 11. CA/TO x EPI & $5,62^{a}(24,96)$ & 14,0 & 3,78 \\
\hline 12. CO/TO $\times \mathrm{EPI}$ & $5,22^{\mathrm{ab}}(21,14)$ & 9,0 & 3,74 \\
\hline FV. & $\mathrm{F}$ & & $p$-value \\
\hline Especie & 8,50 & & 0,000 \\
\hline Adhesivo & 999,84 & & 0,000 \\
\hline Especie:adhesivo & 9,44 & & 0,000 \\
\hline
\end{tabular}

CA: Cariniana domestica; CO: Copaifera paupera; TO: Cedrelinga cateniformis; FV: Fuente de variación. Las medias seguidas de una misma letra en una misma columna son estadísticamente iguales, por la prueba de Tukey al $95 \%$ de probabilidad. Los valores entre paréntesis son los coeficientes de variación en porcentaje. 
Los valores promedio de cizallamiento de las juntas encoladas con adhesivo PVA oscilaron de 0,80 MPa (Cariniana domestica/Copaifera paupera) a 1,69 MPa (Cedrelinga cateniformis), mientras que para el adhesivo EPI fueron de 3,76 MPa (Cariniana domestica/Copaifera paupera) a 5,62 MPa (Cariniana domestica/Cedrelinga cateniformis).

El análisis de variancia de la resistencia de las juntas encoladas al cizallamiento indica que el efecto de la interacción entre las variables especie y el adhesivo utilizado es significativo. Ello se aprecia en la prueba de Tukey, donde se observa que el efecto de la especie no es el mismo sobre el cizallamiento para los distintos adhesivos. La prueba de Tukey muestra que, al utilizar el adhesivo PVA, las juntas encoladas con Cedrelinga cateniformis $(1,69 \mathrm{MPa})$ y Copaifera paupera $(1,28 \mathrm{MPa})$ tienen mayores valores y estadísticamente tienen el mismo cizallamiento, mientras que las otras juntas encoladas estudiadas son estadísticamente iguales entre sí, pero muestran menores valores de cizallamiento.

A diferencia de lo observado al emplear adhesivo PVA, con el adhesivo EPI, no existen diferencias en el cizallamiento entre las especies empleadas. Se debe contar con la excepción de las juntas encoladas al combinar Cariniana domestica/Copaifera paupera $(3,76 \mathrm{MPa})$, la cual reportó significativamente menores resultados de cizallamiento que las juntas encoladas de Cariniana domestica/Cedrelinga cateniformis (5,62 MPa).

En relación a la influencia del adhesivo, se encontró que todos los ensayos de cizallamiento con las juntas encoladas con adhesivo EPI presentan mayor resistencia que las juntas elaboradas con adhesivo PVA, lo cual era esperado, por la baja resistencia del adhesivo PVA al agua, Esto confirma lo mencionado por Iwakiri (2005) y Frihart \& Hunt (2010) sobre la baja resistencia del adhesivo PVA a la humedad, debido a que se fijan por el solvente (agua) y es absorbido por la madera para luego ser liberado a la atmosfera; mientras que el adhesivo EPI, por la reacción de los grupos isocianatos con los grupos hidroxilos de la madera, conforman enlaces duraderos que le dan una alta resistencia al agua y a la temperatura.

Los valores promedio encontrados en todas las juntas encoladas con los adhesivos PVA y EPI fueron inferiores a los encontrados por Bila et al. (2016) para seis especies de maderas tropicales de la Amazonía (Eschweilera coriaceae, Manilkara amazonica, Protium puncticulatum, Inga paraensis, Eschweilera odora y Byrsonima crispa), con masas especificas aparentes mayores de 0,81 $\mathrm{g} / \mathrm{cm}^{3}$, encontrándose valores de 3,14 a 7,43 MPa para el adhesivo PVA y de 7,66 a 13,76 MPa para el adhesivo EPI respectivamente. Sin embargo, los resultados reportados por Iwakiri et al. (2017) para la especie Genipa americana, con masa especifica aparente de $0,74 \mathrm{~g} / \mathrm{cm}^{3}$, fueron inferiores a los obtenidos en el presente estudio para el adhesivo PVA y similares con el adhesivo EPI, hallándose valores de 0,28 MPa con el adhesivo PVA y de 4,20 MPa con el adhesivo EPI. Estos resultados podrían deberse a la mayor masa especifica que poseen las especies arriba mencionadas, propiciando una mayor resistencia en la línea de cola.

Para el porcentaje de falla en la madera, los valores obtenidos fueron de $0 \%$ para todas las juntas encoladas con el adhesivo PVA, confirmándose su baja resistencia al contacto directo con el agua, y de 7,0\% a 21,5\% con el adhesivo EPI, que presenta una mejor resistencia a la humedad, pero se encuentran por debajo del valor mínimo (mayor de 40\%) considerado en la norma UNE-EN 13353 (Asociacion Española de Normalizacion y Certificacion, 2011), que se aplica para especies cuya masa especifica aparente sea igual o menor de $0,6 \mathrm{~g} / \mathrm{cm}^{3}$.

Los valores de falla en la madera encontrados con las juntas encoladas con adhesivo PVA fueron similares a los reportados por Bila et al. (2016) para seis especies tropicales y por Iwakiri et al. (2017) para la Genipa americana, que fueron en ambos casos de 0\%, mientras que para el adhesivo EPI fueron de 3,3\% a 33,3\% y de 3,0\% respectivamente.

Los valores promedio del $5^{\circ}$ percentil inferior para las juntas encoladas con el adhesivo PVA variaron de 0,42 MPa (Cariniana domestica/Copaifera paupera) a 1,26 MPa (Cedrelinga cateniformis); además, para el adhesivo EPI fluctuaron de 2,06 MPa (Cariniana domestica/Copaifera paupera) a 4,22 MPa (Cariniana domestica). Todos los resultados reportados para las juntas encoladas con el adhesivo PVA no cumplen con el requisito mínimo del $5^{\circ}$ percentil inferior de 2,5 MPa indicado en la norma UNE-EN 13353 (Asociacion 
Española de Normalizacion y Certificacion, 2011), mientras que los realizados con el adhesivo EPI, exceptuando la combinación Cariniana domestical Copaifera paupera, sí cumplen con el valor mínimo establecido.

\section{Resistencia de las juntas encoladas al ensayo de cizallamiento- con pretratamiento ambiente húmedo}

Todas las juntas encoladas de las probetas elaboradas con adhesivo PVA se despegaron durante el pretratamiento en agua hirviendo, por lo que sólo se realizó el análisis con las producidas con el adhesivo EPI. Los resultados de los ensayos de cizallamiento, porcentaje de falla en la madera y el $5^{\circ}$ percentil inferior de las juntas encoladas con pretratamiento para ambiente húmedo se indican en la Tabla 5.

Tabla 5. Resultados de los ensayos de cizallamiento con pretratamiento ambiente húmedo/interacciones.

\begin{tabular}{cccc}
\hline Tratamiento & Cizallamiento (MPa) & Falla (\%) & $\begin{array}{c}\mathbf{5}^{\circ} \text { Percentil inferior } \\
\text { (MPa) }\end{array}$ \\
\hline 7. CA $\times$ EPI & $4,28^{\mathrm{ab}}(32,03)$ & 6,8 & 2,57 \\
8. CO $\times$ EPI & $3,31^{\mathrm{b}}(32,73)$ & 4,5 & 2,55 \\
9. TO $\times$ EPI & $3,36^{\mathrm{ab}}(17,18)$ & 9,5 & 2,43 \\
10. CA/CO $\times \mathrm{EPI}$ & $3,18^{\mathrm{b}}(21,27)$ & 3,5 & 1,77 \\
11. CA/TO $\times \mathrm{EPI}$ & $4,65^{\mathrm{a}}(25,11)$ & 7,0 & 3,55 \\
12. CO/TO $\times \mathrm{EPI}$ & $3,12^{\mathrm{b}}(18,85)$ & 7,5 & 1,62 \\
FV. & $\mathrm{F}$ & & $\mathrm{p}$-value \\
Especie & 4,38 & & 0,002 \\
\hline
\end{tabular}

CA: Cariniana domestica; CO: Copaifera paupera; TO: Cedrelinga cateniformis; FV: Fuente de variación. Las medias seguidas de una misma letra en una misma columna son estadísticamente iguales, por la prueba de Tukey al 95\% de probabilidad. Los valores entre paréntesis son los coeficientes de variación en porcentaje.

Los valores promedio de cizallamiento de las juntas encoladas evaluadas fluctuaron de 3,12 MPa (Copaifera paupera/Cedrelinga cateniformis) a 4,65 MPa (Cariniana domestica/Cedrelinga cateniformis), mientras que los porcentajes de falla de la madera fueron de $3,5 \%$ a $9,5 \%$.

Según el análisis de variancia, el efecto de la especie empleada es significativo sobre la resistencia al cizallamiento. La prueba de Tukey, con un nivel de confianza de 95\%, muestra que los resultados de resistencia al cizallamiento de las juntas encoladas de Cariniana domestica/Cedrelinga cateniformis (4,65 MPa) presentan los mayores valores; la Copaifera paupera (3,31 MPa), la Cariniana domestica/Copaifera paupera $(3,18 \mathrm{MPa})$ y la Copaifera paupera/Cedrelinga cateniformis (3,12 MPa) muestran los menores valores, los cuales podrían deberse al mayor contenido de extractivos y $\mathrm{pH}$ que presenta la Copaifera paupera, mientras que las juntas Cariniana domestica (4,28 MPa) y Cedrelinga cateniformis (3,36 MPa) pueden ser consideradas en ambos grupos.

Los resultados encontrados en las juntas encoladas con el adhesivo EPI fueron superiores a los reportados por Almeida (2013), para las maderas tropicales Astronium lecointei $(0,99 \mathrm{MPa})$, Himenaea courbaril $(1,72 \mathrm{MPa})$ y Tabeuia spp (1,30 MPa); estos resultados podrían explicarse por el alto contenido de extractivos que poseen $(11,90 \%$ a $13,02 \%)$ comparados con las especies en estudio $(4,07 \%$ a $7,01 \%)$, lo cual concuerda con lo indicado por Iwakiri (2005) y Gonzales (2013), quienes señalan que la presencia de extractivos en exceso o extractivos apolares, así como las condiciones de la superficie a encolar pueden perjudicar su humectación por el adhesivo, ya que reducen la higroscopicidad y permeabilidad, produciendo una menor resistencia en el encolado.

Los valores alcanzados de porcentaje de falla en la madera fueron de 3,5\% a 9,5\%, que son menores al reportado por Fonte (2016) para la especie Cryptomeria japónica, que fue de 18\%, y mayores a lo obtenido por Lau (2017) para la especie Populus deltoides, que fue de 0\%

Los valores promedio hallados del $5^{\circ}$ percentil inferior de la resistencia al cizallamiento para las juntas encoladas fluctuaron de 1,62 MPa a 3,55 MPa, encontrándose que las 
probetas de Cariniana domestica, Copaifera paupera y Cariniana domestica/Cedrelinga cateniformis cumplen con el valor mínimo del $5^{\circ}$ cuartil inferior de 2,5 MPa indicado en la norma UNE-EN 13353 (Asociacion Española de Normalizacion y Certificacion, 2011). Estos resultados confirman lo señalado por Frihart \& Hunt (2010) y Frihart (2013), quienes afirman que el adhesivo EPI le suministra una alta resistencia a la humedad y temperatura.

\section{CONCLUSIONES}

Las especies estudiadas son categorizadas, por su masa especifica aparente, como semipesadas (Copaifera paupera y Cedrelinga cateniformis) y pesada (Cariniana domestica); además, muestran un contenido de humedad dentro del rango aceptado para los tipos de adhesivos evaluados.

El contenido de extractivos y pH se ubican dentro de los rangos adecuados reportados en la literatura, encontrándose que la especie Copaifera paupera presentó mayores valores en las dos variables evaluadas.

En los ensayos de cizallamiento con los tres pretratamientos aplicados, el adhesivo EPI brindó un mejor comportamiento de resistencia en las juntas encoladas estudiadas, en comparación con el adhesivo PVA.

Los valores promedio de resistencia al cizallamiento de todas las juntas encoladas con los adhesivos PVA y EPI sin pretratamiento, prueban su aptitud para la producción de paneles EGP para uso en interiores, recomendándose el adhesivo PVA para reducir los costos de fabricación.

Los resultados de los ensayos de cizallamiento de las juntas encoladas con el adhesivo EPI con pretratamiento en ambiente seco, exceptuando la combinación Cariniana domestica/Copaifera paupera, cumplen con el valor mínimo del $5^{\circ}$ percentil inferior de 2,5 MPa señalado en la norma UNE-EN 13353:2011 (Asociacion Española de Normalizacion y Certificacion, 2011).

Los ensayos de resistencia al cizallamiento de las juntas encoladas con adhesivo EPI de las especies Cariniana domestica, Copaifera paupera y la Cariniana domestica/Copaifera paupera, demuestran su aptitud para la producción de paneles EGP para uso en ambiente húmedo.

\section{REFERENCIAS BIBLIOGRAFICAS}

Almeida, C. C. F. (2015). Avaliação da qualidade da colagem da madeira de Cupressus lusitanica Mill. para a produção de painéis colados lateralmente (EDGE GLUED PANEL - EGP) (Dissertação de mestrado). Centro de Ciências Agroveterinárias, Universidade do Estado de Santa Catarina, Lages.

Almeida, V. C. (2013). Avaliação do potencial de uso de resíduos de madeira tropical para produção de painéis colados lateralmente - EGP (Tese de doutorado). Universidade Federal de Paraná, Curitiba.

Asociacion Española de Normalizacion y Certificacion - AENOR. (1978). UNE 56-540. Características físicomecánicas de la madera. Interpretación de los resultados de los ensayos. Madrid: AENOR.

Asociacion Española de Normalizacion y Certificacion - AENOR. (1995). UNE-EN 326-1. Tableros derivados de la madera. Muestreo, despiece e inspección. Parte 1. Muestreo y despiece de probetas y expresión de resultados de ensayo. Madrid: AENOR.

Asociacion Española de Normalizacion y Certificacion - AENOR. (2007). UNE-EN 314-1. Tableros contrachapados. Calidad de encolado. Parte 1: Método de ensayo. Madrid: AENOR.

Asociacion Española de Normalizacion y Certificacion - AENOR. (2009). UNE-EN 13354. Tableros de madera maciza. Calidad de encolado. Método de ensayo. Madrid: AENOR.

Asociacion Española de Normalizacion y Certificacion - AENOR. (2011). UNE-EN 13353:2009+A1. Tableros de madera maciza (SWP). Requisitos. Madrid: AENOR.

Bila, N. F., Iwakiri, S., Trianoski, R., \& Prata, J. G. (2016). Avaliação da qualidade de juntas coladas de seis espécies de madeiras tropicais da Amazônia. Floresta, 46(4), 455-464.

Fonte, A. P. N. (2016). Utilização da madeira de Cryptomeria japonica para a produção de painéis colados lateralmente e aplicação de acabamento superficial (Dissertação de mestrado). Setor de Ciências Agrárias, Universidade Federal do Paraná, Curitiba. 
Frihart, C. R. (2013). Wood adhesion and adhesives. In R. M. Rowell. Handbook of wood chemistry and wood composites (chap. 9, 2. ed., 669 p.). Boca Raton: CRC Press. http://dx.doi.org/10.1201/b12487.

Frihart, C. R., \& Hunt, C. G. (2010). Adhesive with wood materials bond formation and performance. In Forest Products Laboratory. Wood handbook-wood as an engineering material (chap. 10, 508 p.). Madison: Forest Products Laboratory.

Gonzales, H, E. (2013). Transformación química de la madera (129 p.). Lima: Universidad Nacional Agraria La Molina.

Held, C. H., Pawlowski, G., Paredes, A., \& Calo, I. (2015). Cadenas de valor en el sector forestal del Perú. Informe diagnóstico y desarrollo estratégico (106 p.). Freiburg: Global Green Growth Institute.

Instituto Nacional de la Calidad - INACAL. (2014a). NTP 251.010. Madera. Método para determinar el contenido de humedad. Lima: INACAL.

Instituto Nacional de la Calidad - INACAL. (2014b). NTP 251.011. Madera. Método para determinar la densidad. Lima: INACAL.

Iwakiri, S. (2005). Painéis de madeira reconstituída (243 p.). Curitiba: FUPEF.

Iwakiri, S., Campelo, S., Trianoski, R., \& Aguiar, O. R. (2017). Utilização da madeira de Genipa americana para produção de painéis de colagem lateral EGP. Floresta, 47(1), 129-135. http://dx.doi.org/10.5380/rf.v47i1.48453.

Iwakiri, S., Trianoski, R., Fonte, A. P. N., França, M. C., Lau, P. C., \& Molleken, R. (2016). Potencial de uso de madeiras de Dinizia excelsa Ducke e Protium puncticulatum J.F. Macbr para produção de painéis EGP. Scientia Forestalis, 44(111), 709-717. http://dx.doi.org/10.18671/scifor.v44n111.17.

Iwakiri, S., Trianoski, R., França, R. F., Gonçalves, T. A. P., Loiola, P. L., Campelo, S. R., \& Farias, S. M. A. P. (2015). Avaliação da resistência de juntas colada da madeira de Cryptomeria japônica e Sequoia sempervirens com diferentes adesivos. Scientia Forestalis, 43(105), 19-26.

Lau, P. M. (2017). Produção de painéis de colagem lateral - EGP com madeira de Populus deltoides (Dissertação de mestrado). Setor de Ciências Agrárias, Universidade Federal do Paraná, Curitiba.

Pizzi, A., \& Mittal, K. L. (2003). Handbook of adhesive technology. New York: Marcel Dekker.

Thechnical Association of the Pulp and Paper Industry - TAPPI. (2016). TAPPI T 252 - om-16: pH and electrical conductivity of hot water extracts of pulp, paper, and paperboard. Atlanta: TAPPI.

Thechnical Association of the Pulp and Paper Industry - TAPPI. (2017). TAPPI T 204 - cm-17: Solvent extractives of Wood and pulp. Atlanta: TAPPI.

Contribuciones de los autores: JCCR: Conceptualización, Curaduría de Datos, Análisis Formal, Investigación Metodología, Validación, Escritura - Primera Redacción; SI: Conceptualización, Metodología, Escritura - Revisión y Edición; RT: Conceptualización, Metodología, Escritura - Revisión y Edición; HEGM: Conceptualización, Análisis Formal, Metodología, validación. 